\title{
Metasurfaces as energy valves for sustainable energy management
}

\author{
YOSHIAKI NishIJIMA, ${ }^{1,}{ }^{*}$, SYUNYA KIMURA, ${ }^{1}$ YU TAKESHIMA, ${ }^{1}$ AND \\ SAULIUS JUODKAZIS ${ }^{2,3,4}$ \\ ${ }^{1}$ Department of Electrical and Computer Engineering, Graduate School of Engineering, Yokohama \\ National University, 79-5 Tokiwadai, Hodogaya-ku, Yokohama, 240-8501, Japan \\ ${ }^{2}$ Optical Sciences Centre and ARC Training Centre in Surface Engineering for Advanced Materials \\ (SEAM), School of Science, Swinburne University of Technology, Hawthorn, VIC 3122, Australia \\ ${ }^{3}$ Institute of Advanced Sciences, Yokohama National University, 79-5 Tokiwadai, Hodogaya-ku, Yokohama \\ 240-8501, Japan. \\ ${ }^{4}$ Tokyo Tech World Research Hub Initiative (WRHI), School of Materials and Chemical Technology, Tokyo \\ Institute of Technology, 2-12-1, Ookayama, Meguro-ku, Tokyo 152-8550, Japan \\ *nishijima-yoshiaki-sp@ynu.ac.jp
}

\begin{abstract}
Control of light absorption and transmission by metal-insulator-metal (MIM) metasurfaces are important for applications in optical windows. In this study we show realization of photo-thermal energy conversion for the radiative cooling. By controlling absorption and transparency of MIM metasurfaces with thin metal substrate and Indium-Tin-Oxide (ITO) substrates, a high transparency at visible wavelengths and high absorption at mid-infrared wavelengths was realized for a prospective application in efficient cooling or heating of the room.
\end{abstract}

\section{Introduction}

Metasurfaces are increasingly investigated for applications of efficient photo-thermal energy conversion [1-22]. Especially appealing is application for the sky radiator, a solution for cooling a room through optical windows exploiting high transparency of air at 8-13 $\mu \mathrm{m}$ mid-IR spectral range. Typical metasurfaces for absorption control are made using a MIM layout of a Metal base plate, Insulator and Metal nanostructures. Thick $(\sim 200 \mathrm{~nm})$ metal base plate prevent the optical transmission at any wavelength. The concept of radiation cooling is that the thermal energy generated in the room can be emitted by a metasurface at the atmospheric IR transparency window. In these applications, metasurfaces are assumed to use a transparent (at visible) window or paint materials compatible with use in practical building/architecture solutions. Especially for the optical window applications, it is important to have properties of transparency at visible wavelengths while keeping a blocking (absorption and reflection) capability at the mid-infrared (MIR) spectral range.

Another useful complimentary functionality of thermal management in a building would be realisation of radiative heating, by enhancing the green house effect inside the room via control of the radiation wavelength at the air absorption band (rather transmission used for radiative cooling).

Metasurfaces are very promising materials enabling an easy control of their absorption and radiation wavelength via geometrical structure of MIM configurations. Ideally, by control of heating and cooling with passive devices, it should be possible to create comfortable conditions in the room without electric energy consumption. In agriculture, passive control of greenhouse conditions are required for stable crop harvesting. Need of such technology will be exacerbated due to climate change. The concept of radiative heating and cooling by metasurface window is shown in Fig. 1(a) with a transmission valve metasurfaces shown in Fig. 1(b).

In this study we demonstrate MIM structures for combined functionality of visible wavelength 


\section{(a)}
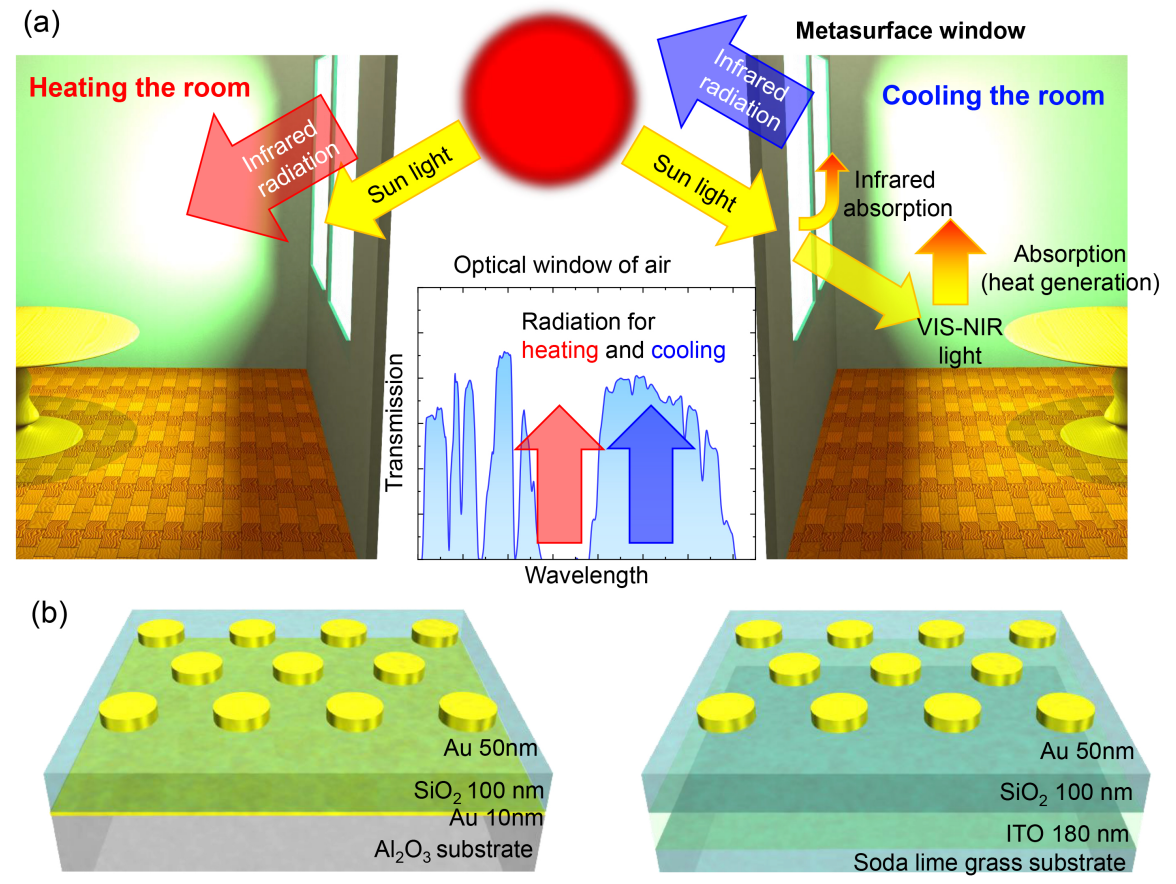

Fig. 1. Schematic illustration of a passive control of energy flow in a visible and near-IR transparent (for the radiative cooling) as well as for MIR absorptive metasurface to control radiative heating. (b) Design of required metasurfaces investigated in this study.

transparency with MIR absorption for applications in optical windows via following designs based on: 1) thin Au film ( $10 \mathrm{~nm}$ thickness) for metal ground plate (Thin-Au) and 2) Indium Tin Oxide (ITO) as the metal ground plate [23-36]. The first design is adopted for windows with radiative cooling of room and the second for room heating (a greenhouse effect).

\section{Experimental procedures and modeling}

\subsection{Fabrication of transparent metasurfaces}

Metasurfaces were fabricated with the standard electron beam lithography (EBL). Both Au thin film and ITO substrates were prepared via following route. Au thin film has vacuum evaporated on a sapphire $\left(\mathrm{Al}_{2} \mathrm{O}_{3}\right)$ substrate with $2 \mathrm{~nm}$ of $\mathrm{Cr}$ as an adhesion layer. Then, a $100 \mathrm{~nm}$ of $\mathrm{SiO}_{2}$ film has deposited using electron beam (EB)-evaporation without any adhesion layer. ITO substrates were commercially acquired with $180 \mu$ m thickness. They had $\sim 6 \Omega / \square$ sheet resistance, which showed $10^{5}$ to $10^{6}(\Omega . \mathrm{cm})^{-1}$ conductivity on the soda-lime glass substrate. A film of $100 \mathrm{~nm}$ of $\mathrm{SiO}_{2}$ has deposited using EB-evaporation without any adhesion layer.

For both substrates, EBL was used to define $300 \mathrm{~nm}$ to $2000 \mathrm{~nm}$ diameter nanodisc patterns. Then, a $50 \mathrm{~nm}$ of Au with $2 \mathrm{~nm} \mathrm{Cr}$ was evaporated. The following lift-off was performed by dip into an organic solvent, N-Methyl-2-pyrrolidone (NMP) heated up to $100^{\circ} \mathrm{C}$ until the metal-on-resist layer was floated. Then, samples were washed in acetone and methanol, dried by air blowing resulting in final transparent MIM windows (Fig. 1(b)) used for characterisation. 


\subsection{Optical characterization}

Optical transmission and reflection spectra were measured using microscopy-based spectroscopic techniques. At visible wavelengths, an upright microscope (Olympus BX-63) with combined spectrometer and cooled CCD (SP2150 and PIXIS 256E, Teledyne Princeton instruments) was implemented. For MIR wavelengths, FT-IR (FTIR-6600, JASCO) with microscope accessory (IRT-1000, JASCO) was used. The reference of transmission and reflection was a broad-band reflection mirror (100\% from $400 \mathrm{~nm}$ to $2000 \mathrm{~nm}$ ) and Au-mirror (98\% at MIR wavelengths). Air ambient was used for the transmission measurements.

\subsection{FDTD simulation of MIM properties}

Finite difference time domain (FDTD) simulations were performed with a home made workstation with Dual CPU (AMD EPYC 730216 core $\times 2$ ), 512 GB of DDR4 memories. For the spectrally refined modeling, a high accuracy simulations were performed to reveal detailed spectral features. Commercial software FDTD solution (ANSYS/Lumerical) was used for numerical modeling. For a wide wavelength region from visible to MIR, the Palik's optical permittivity data were used for $\mathrm{Au}, \mathrm{Cr}, \mathrm{SiO}_{2}, \mathrm{Al}_{2} \mathrm{O}_{3}$. For the ITO properties which were difficult to measure experimentally over entire vis-MIR spectral range, we setup a model based on the 3D conductive and plasma based on Drude models described next. For the Drude's free electron model in two equivalent presentations:

$$
\begin{gathered}
\varepsilon(\omega)=\varepsilon_{\mathrm{DC}}+i \frac{\sigma_{\mathrm{DC}}}{\omega \varepsilon_{0}}, \\
\varepsilon(\omega)=\varepsilon_{\infty}-\frac{\omega_{\mathrm{p}}}{\omega^{2}+i \omega \Gamma},
\end{gathered}
$$

where $\varepsilon(\omega)$ is the frequency dependence of permittivity, $\varepsilon_{\mathrm{DC}}$ is permittivity of ITO at the direct current (DC) conditions, $\sigma$ is the conductivity, $\varepsilon_{0}$ is the permittivity of vacuum, $\varepsilon_{\infty}$ is the permittivity at high frequency, $\omega_{\mathrm{P}}$ is the plasma frequency, $\Gamma$ is the damping constant. Wolf et. al [37] analyzed ITO films using Drude model and found the parameters for the sputtered films to be following $\varepsilon_{\infty}=4.4, \omega_{\mathrm{P}}=3.36 \times 10^{16} \mathrm{rad} / \mathrm{s}, \Gamma=1.05 \times 10^{14} 1 / \mathrm{s}$.

The 3D conductivity model can be used for materials which have low losses. A simple formula for conductivity at direct current (DC) condition can be derived to define model from DC measurements of refractive index and conductivity. However, if material has a frequency(wavelength) dependence of electrical conductivity, it is obvious that a different model has to be applied for simulations. If free electric charges can follow at the AC frequency, the 3D conductive model becomes a suitable approximation. However, another model is required if response of charges to a high frequency is not instantaneous. The Drude model is promising for modeling the free electron behaviour in noble metals. It was successfully adapted for plasmonic metals $\mathrm{Au}, \mathrm{Ag}, \mathrm{Cu}$. The relationship between parameters of the 3D conductivity and Drude-plasma can be combined as:

$$
\sigma_{D C}=\left(\frac{N}{V}\right)\left(\frac{e^{2} \tau}{m^{*}}\right)=d e \mu=\tau \omega_{p}^{2}
$$

where $d$ is the density of electrons $N / V$ (i.e., Number $/$ Volume), $\mu$ is their mobility and $\mathrm{m}^{*}$ is the effective mass. In the Drude model of free electrons, $\tau$ is the relaxation time with $\tau=1 / \Gamma$. ITO films that have a high carrier (electron) density and high mobility can be described by the high $\omega_{\mathrm{P}}$, large permittivity and longer relaxation time $\tau$. 

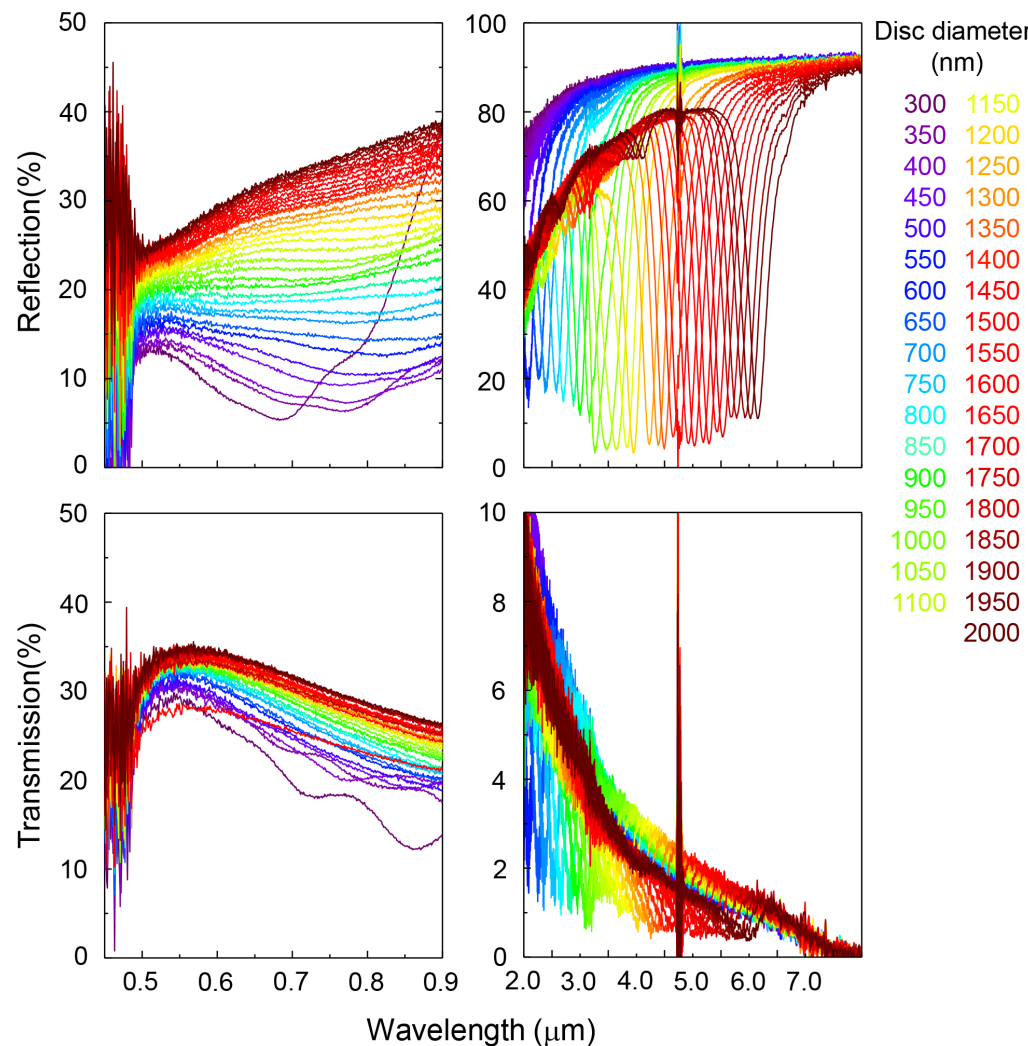

Fig. 2. Experimental spectra. Optical reflection and transmission spectra of thin-Au metasurfaces (Fig. 1(b)) from visible to MIR wavelengths. Thicknesses of layers in the MIM structure: Au-disc of $50 \mathrm{~nm}, \mathrm{SiO}_{2}$ of $100 \mathrm{~nm}$ and Au film of $10 \mathrm{~nm}$ (with $2 \mathrm{~nm}$ of $\mathrm{Cr}$ adhesion layer); substrate was $0.3 \mathrm{~mm}$ sapphire. The period - to - diameter ratio is 2 . A spectrally narrow anti-reflective band $(R \rightarrow 0)$ at IR wavelengths with simultaneous low transmission $(T \rightarrow 0$ ) marks position of strong absorption (an expected strong thermal emission for the radiative cooling).

\section{Results and Discussion}

\subsection{Au as a metal base in MIM metasurface}

Figure 2 shows the optical reflection and transmission spectra within visible to near-IR and near-IR to MIR wavelengths regions, respectively. A low reflectance $R$ was observed for the thin-Au MIM samples. The reflective properties of metasurface can be explained by coupling between plasmon resonance of metal nanodiscs and induced plasmon resonance of the metal ground plate. If the thickness of metal ground plate become thinner than the skin depth in the metal (at that wavelength), the induced plasmon resonance at $\mathrm{Au}-\mathrm{SiO}_{2}$ interface is expected. Then a re-radiation forward is expected from metasurface at such conditions. Such feature was recognisable in the transmittance $T$ spectra as well. Transmission was well suppressed to less than $10 \%$ and their reflectance spectra show strong anti-reflection properties.

However, in the visible wavelengths region, transmission became less than $40 \%$ in air. Au has a $\omega_{\mathrm{P}}$ of $13.8 \times 10^{16} \mathrm{rad} / \mathrm{s}$, which corresponds to the $136 \mathrm{~nm}$ wavelength. When the frequency of light becomes larger than the $\omega_{\mathrm{P}}$, free electron cannot follow E-field of light and metal 


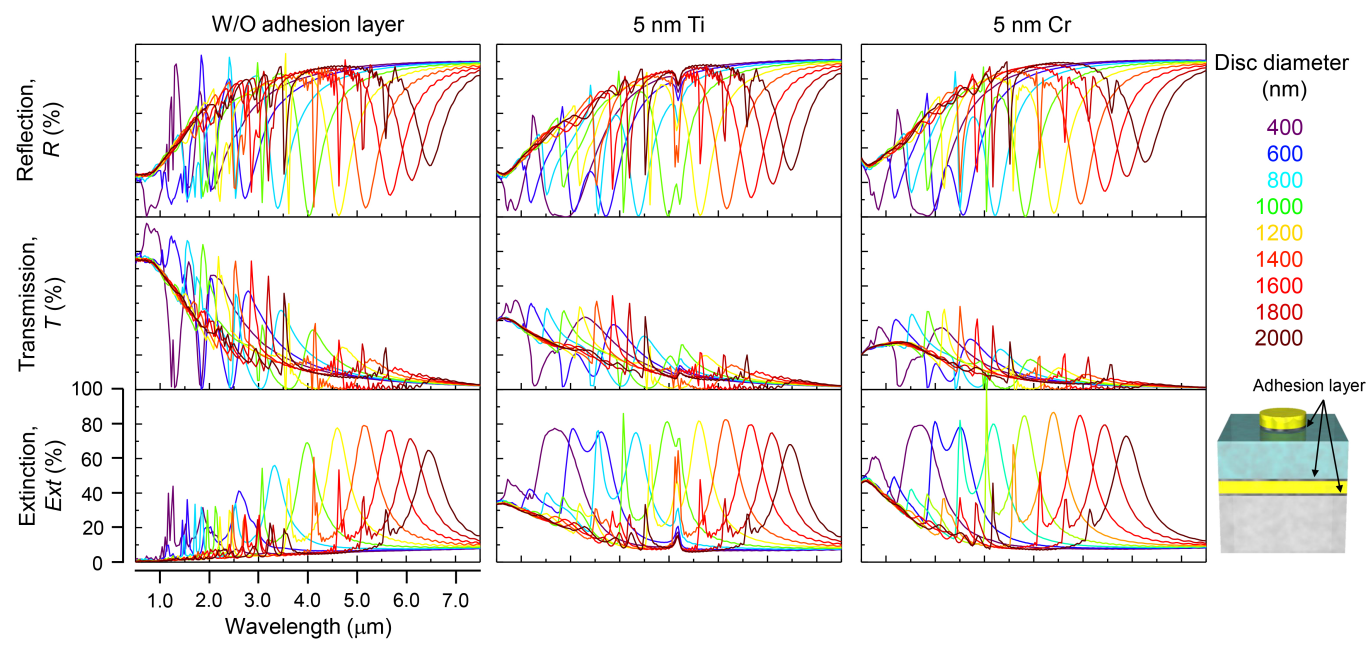

Fig. 3. Influence of an adhesion nano-layer. FDTD simulations for thin-Au MIM metasurfaces without adhesion layer, with $5 \mathrm{~nm}$ Ti and with $5 \mathrm{~nm} \mathrm{Cr}$ as adhesion layers. Calculations are made for the MIM structure only without substrate.
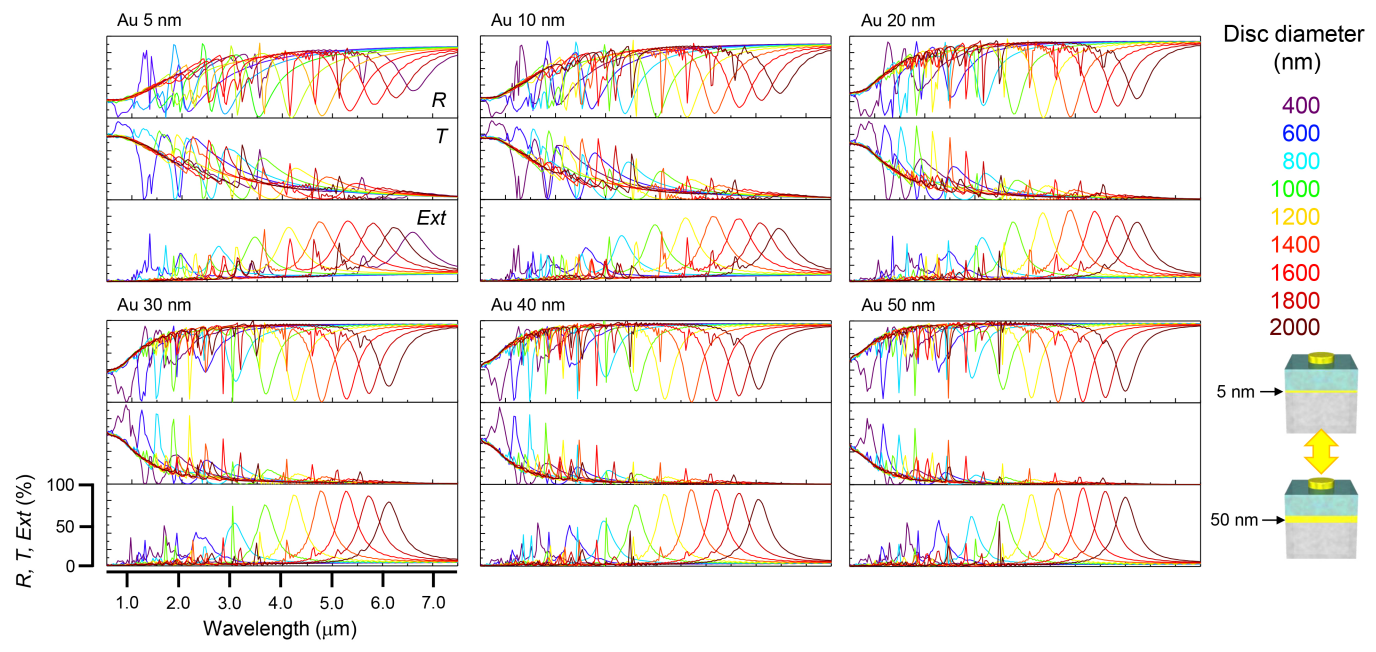

Fig. 4. Influence of thickness of the bottom mirror in MIM. FDTD simulations for different thickness thin-Au MIM metasurface without adhesion layers.

becomes endowed with dielectric-like property. The $\mathrm{Au}, \mathrm{Ag}, \mathrm{Cu}$ have similar $\omega_{\mathrm{P}}$ at 13.8, 14.0, $13.4 \times 10^{15} \mathrm{rad} / \mathrm{s}$, respectively, and at the visible spectral range spectral(color) differences in $R$ and $T$ of those metals mainly comes from the damping constant differences. When the damping constant $\Gamma$ became smaller (Au 1.07, Ag 0.322, Cu $1.45 \times 10^{14} 1 / \mathrm{s}$ ), the high reflectance region extends until the $\omega_{\mathrm{P}}$. Therefore, reflectance of $\mathrm{Au}$ and $\mathrm{Cu}$ at visible wavelength starts decline into the red-to-near-IR wavelength range and it cause an increase in the visible transmission. It would be expected that for $\mathrm{Cu}$, a high transmission region would extend until longer wavelengths than for $\mathrm{Au}$. However, due to a chemical instability of $\mathrm{Ag}$ and $\mathrm{Cu}$, it is difficult to fabricate non-oxided stable thin metal film of pure metal. Therefore, $\mathrm{Au}$ is the more promising material for the application of thin $\sim 10 \mathrm{~nm}$ thickness film for the required optical transmission. However 
(a)

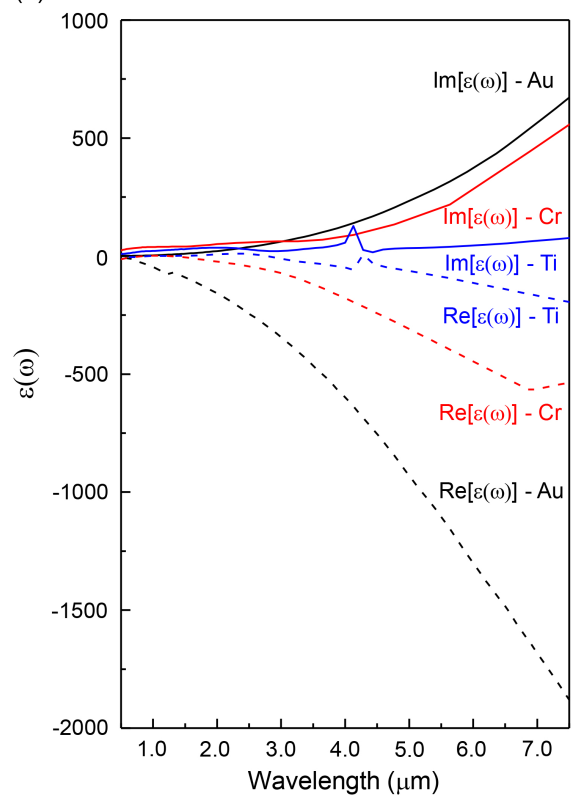

(b)

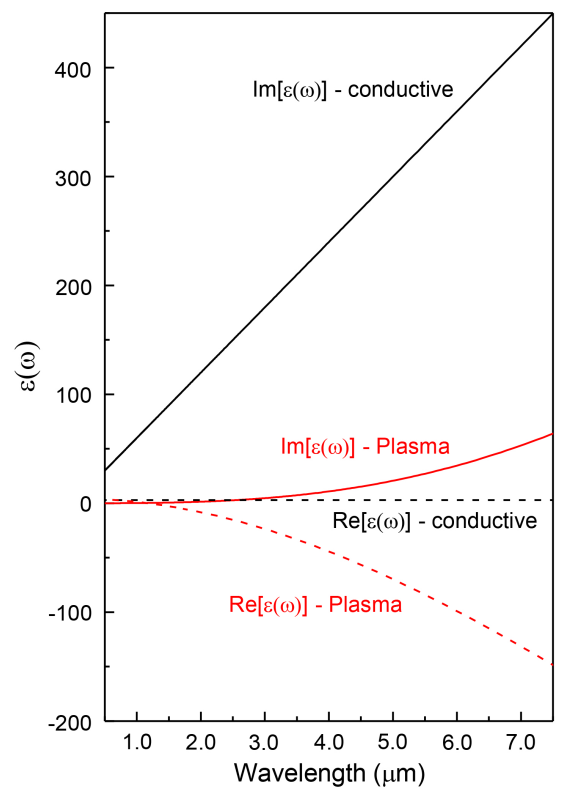

Fig. 5. Optical permittivity of materials that was used in FDTD simulations: (a) Au, Cr, Ti that obtained from Palik's database references. (b) Basic function implemented in the FDTD software for the 3D conductive and plasma (Drude) models with parameters of $\varepsilon_{\mathrm{DC}}=2.94, \sigma=1.0 \times 10^{6}(\Omega \mathrm{cm})^{-1}, \varepsilon_{\infty}=4.4, \omega_{\mathrm{P}}=3.36 \times 10^{16} \mathrm{rad} / \mathrm{s}, \Gamma=1.05 \times$ $10^{14} 1 / \mathrm{s}$.

Au has not enough transparency for some practical applications as a window. For the window application, it is imperative to have transmission beyond $70 \%$.

To identify the origin of optical losses, we have performed FDTD simulations for different adhesion $\mathrm{Cr}$ and Ti films as well as for different Au thicknesses. The results are shown in Fig. 3 and Fig. 4. When there was no adhesion layer (Fig. 3), a film of $10 \mathrm{~nm}$ of gold kept transparency above $80 \%$ at visible wavelengths. However when the adhesion layer of $5 \mathrm{~nm} \mathrm{Cr}$ or Ti has added between metal and dielectric layer, the transmittance $T$ drastically decreased to less than $40 \%$. The effect of Au ground plate thickness has simulated and is shown in Fig. 4 for all range of MIM metasurfaces with different nanodisk diameters. Interestingly, with an increasing thickness of $\mathrm{Au}$, the transmission was still kept above $40 \%$. It was promising that even at $30 \mathrm{~nm} \mathrm{Au} \mathrm{film,}$ $T \approx 80 \%$.

In discussions of experimental data, it is often argued that $2 \mathrm{~nm}$ of $\mathrm{Cr}$ have enough adhesion strength for successful lift-off. Thinner Au at less than $10 \mathrm{~nm}$ would be expected to form an island film rather than an uniform coating. Therefore, a $2 \mathrm{~nm}$ of $\mathrm{Cr}$ and $10 \mathrm{~nm} \mathrm{Au}$ defines the lowest limitation for experimental fabrication. Therefore the transparency of $40 \%$ that has been obtained in experiments is almost the limitation using $\mathrm{Au}$ as the ground plate in MIM metasurfaces. Figure 5(a) shows optical permittivity of $\mathrm{Au}, \mathrm{Cr}$ and $\mathrm{Ti}$ that has obtained from Palik's database and used for the FDTD simulations.

\subsection{ITO as a metal base in MIM metasurface}

Due to the limitation of Au discussed above, it is imperative to use a transparent conductive oxide (TCO) material such as ITO, FTO (F-doped $\mathrm{SnO}_{2}$ ), AZO (Al-doped $\mathrm{ZnO}$ ) and the like. ITO is one of the most popular and commonly used TCO. ITO becomes a key material for the 

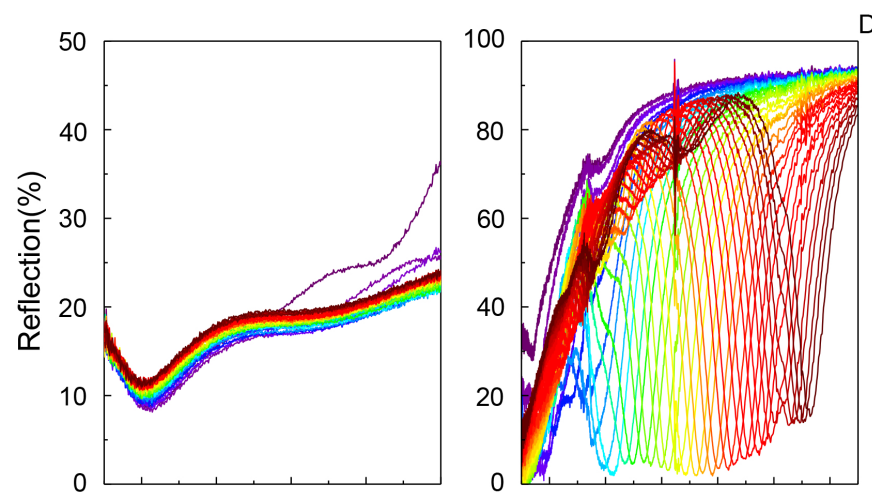

Disc diameter

(nm)

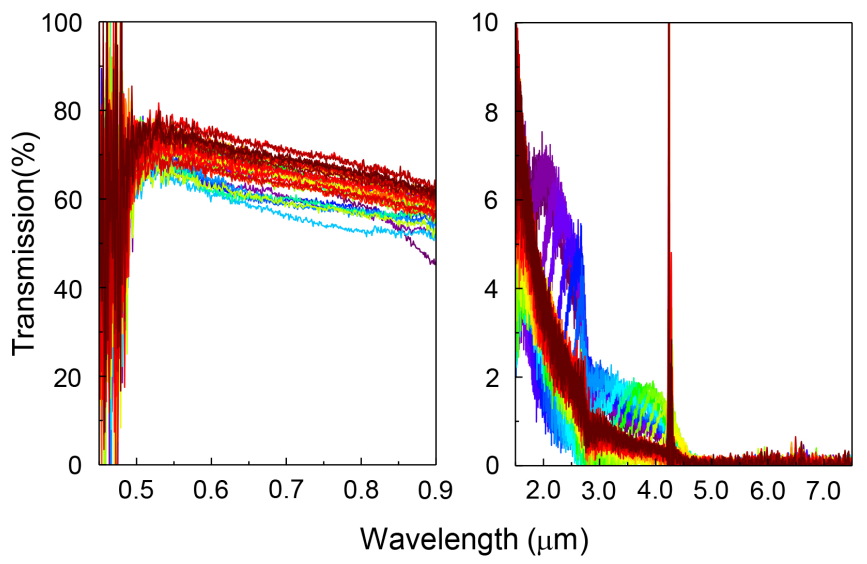

9501800

0001850

10501900

1001950

2000

Fig. 6. Experimental spectra. Optical reflection and transmission spectra of ITO-base MIM metasurfaces made on a soda lime glass.

recent all dielectric metasurfaces. The optical properties of metasurface fabricated using ITO as the ground plate in MIM metasurfaces is shown in Fig. 6. It shows a possibility to realize $80 \%$ transparency at visible wavelengths. In the actual fabrication, ITO was sputtered onto a soda lime glass plate and there was no transparency of at longer than $4 \mu \mathrm{m}$ wavelengths due to the absorption of the glass substrate. However, the plasmon resonance assists the frequency selective anti-reflective performance (absorption) at the resonance wavelength. The reflection spectra show the anti-reflection resonance band which is similar to the Au ground plate in MIM metamaterials discussed above. It is highly expected that selective thermal radiation at those MIR wavelength can be utilised for a greenhouse effect, i.e., a window facilitating warming up of the room.

Further insights in expected optical performance of ITO-base MIM metasurfaces can be gained from numerical modeling. For the FDTD simulation, a model of ITO permittivity is essential. In most of TCO works, conductivity and refractive index are discussed. However the conductivity itself is not a constant to the AC conditions encountered at the optical applications, i.e., the high frequency of light is not represented by the DC conductivity. Therefore it is important to consider the Drude model, which is well established for modeling metallic response or free electron behavior at high frequency. By comparison of both models, the conductive and plasma, FDTD simulations of reflection $(R)$, transmission $(T)$, Extinction $(1-(R+T))$ are summarised in Fig. 7. The optical properties of metasurfaces would expected to contain not only the absorption but also the scattering in the $1-(R+T)$ [38]. As mentioned before, the conductive model cannot 

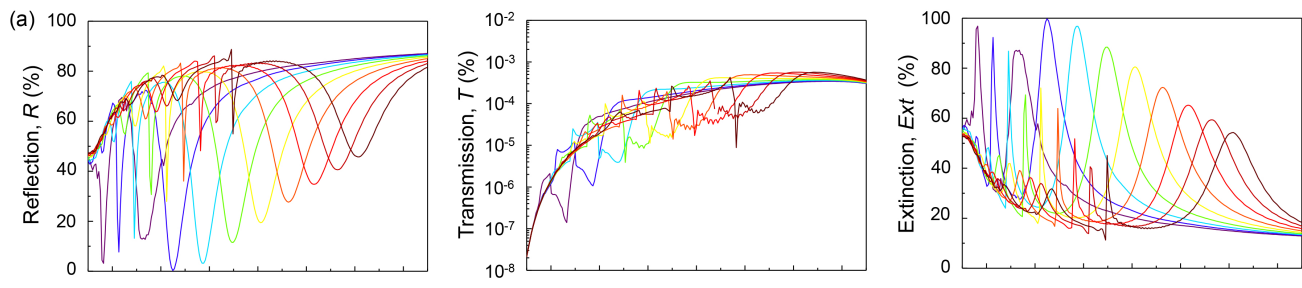

Disc diameter
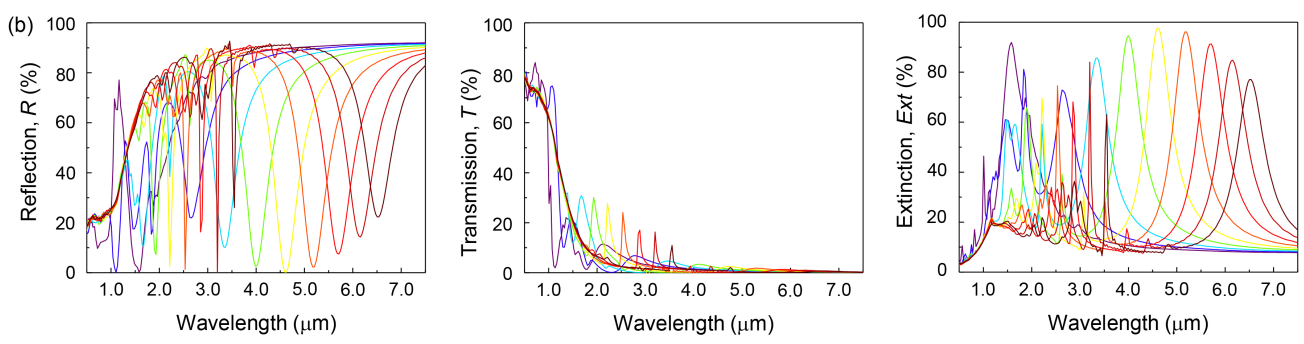

1400

1600

1800

2000

Fig. 7. Reflectance $R$, Transmittance $T$ and Extinction $E x t=1-(R+T)$ spectra. FDTD simulations for ITO metsurfaces with: (a) $3 \mathrm{D}$ conductive model with $\varepsilon_{\mathrm{DC}}=$ 2.94, $\sigma=1.0 \times 10^{6}(\Omega \mathrm{cm})^{-1}$, and (b) the plasma model with $\varepsilon_{\infty}=4.4, \omega_{\mathrm{P}}=3.36 \times$ $10^{16} \mathrm{rad} / \mathrm{s}, \Gamma=1.05 \times 10^{14} 1 / \mathrm{s}$.

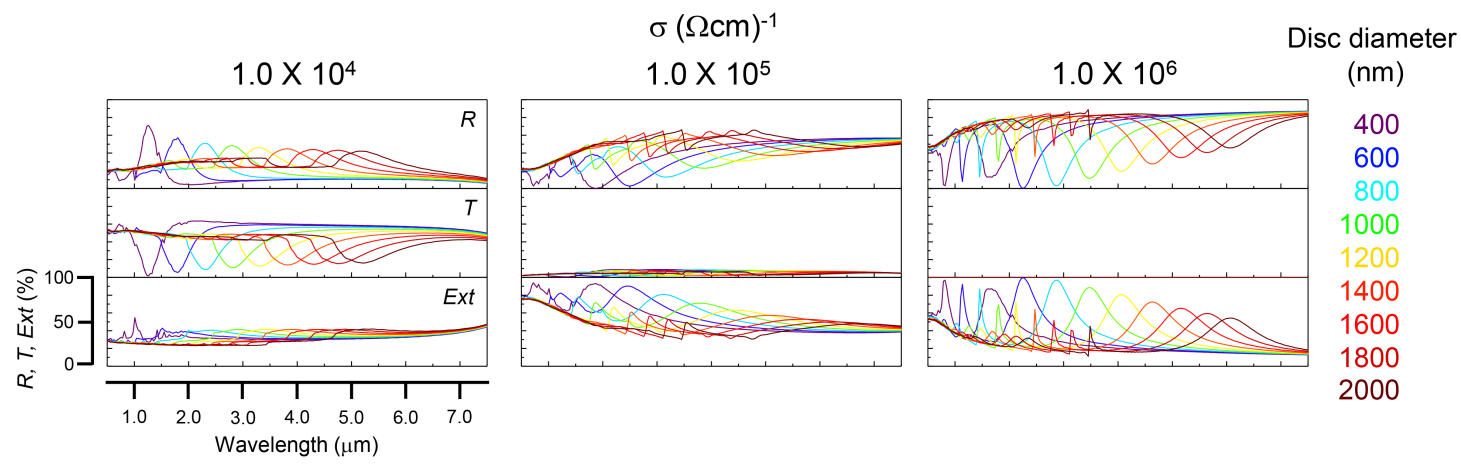

Fig. 8. FDTD simulations for the 3D conductive model with $\varepsilon_{\mathrm{DC}}=2.94$ and $\sigma$ is from $1.0 \times 10^{4}$ to $1.0 \times 10^{6}(\Omega \mathrm{cm})^{-1}$.

reproduce well the experimental results, especially, at the range of anti-reflective resonances and it is limited to the $2-4 \mu \mathrm{m}$ spectral window. Transmission in the entire modeled wavelength region became almost zero.

However, by using the Drude model, the FDTD results became well matching the experimental data. Figures 8 and 9 show various parameter dependencies set for the both models: 1) conductive (Fig. 8) and plasma (Fig. 9). In the transparent conductive films, $\omega_{\mathrm{P}}$ increases when the conductivity $\sigma$ is larger by increasing the carrier density $d$. Therefore, the absorption $\alpha$ becomes stronger and transparency decreases. Therefore, the mobility $\mu$ is increased to increase the conductivity $\sigma$ to match the experimental observation. When electric conductivity of ITO was increased, the absorption losses especially at the MIR wavelengths were increased too.

In the case of gold base plate in MIM, the transmittance was about 40\%. If the thickness of gold was less than $5 \mathrm{~nm}$, an island structure will be formed and the film will not be uniform. This is the reason that $5 \mathrm{~nm}$ Au cannot serve as the base plate in MIM. However, on a substrate such 


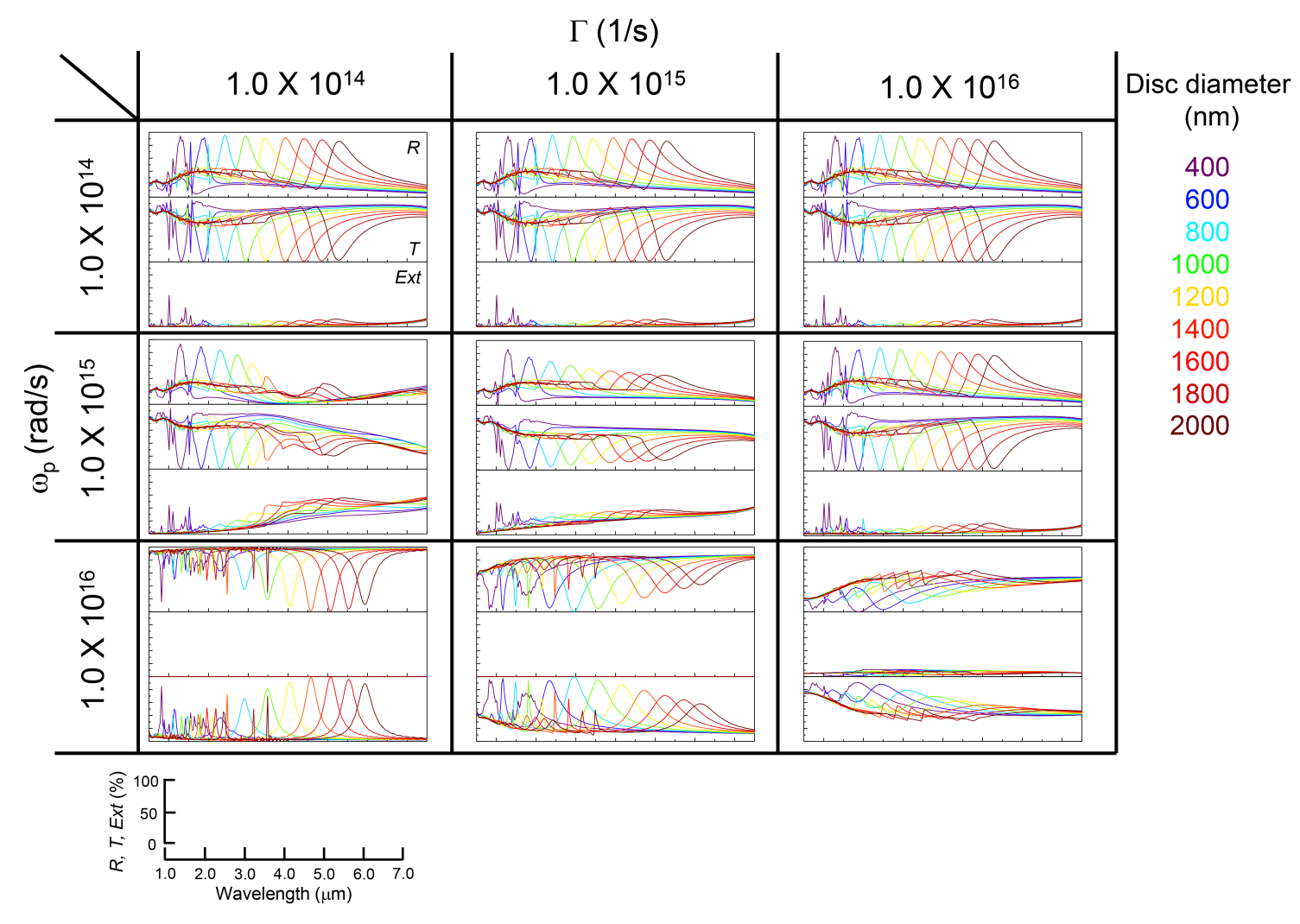

Fig. 9. A series of FDTD simulations for the plasma model with $\varepsilon_{\infty}=4.4, \omega_{\mathrm{P}}$ was changing from $1.0 \times 10^{14}$ to $1.0 \times 10^{16} \mathrm{rad} / \mathrm{s}, \Gamma$ is from $1.0 \times 10^{14}$ to $1.0 \times 10^{16} 1 / \mathrm{s}$.

as mica, a flat curtain can be obtained at the atomic level of gold [39], so it is thought that further thinning of gold can be realized. In the case of gold, it is necessary to place about $4 \mathrm{~nm}$ of $\mathrm{Cr}$ as an adhesive layer for each layer of dielectric, which cause large losses in light transmission. In addition, the experimental requirement for gold to have a film thickness of $10 \mathrm{~nm}$ will also lead to reduction in transmittance of visible light.

Due to the tail of plasmon resonance in MIR wavelengths, transmission and reflection spectra varied with the disc diameter as shown in Figs. 7,8. Especially the smaller disc diameters reduces the transmission at visible wavelengths. On the other hand, the optical properties of ITO show the resonance wavelength shift towards the longer wavelengths. This is due to the fact that ITO has a larger real part of the refractive index. Therefore the effects of reduced $T$ extending to the visible wavelength region is smaller than that for the Au thin mirror MIM structures.

Let us further explore which transparent substrates are most promising for control of $R \& T$ at the visible and MIR spectral window. The transmission optical window of sapphire is down to $5 \mu \mathrm{m}$ in IR, while the absorbance of soda lime glass starts at a shorter that $2.8 \mu \mathrm{m}$ wavelength. This absorption limitation appeared in the transmission spectra in MIR. If there is no absorption by the substrate, it would be expected to observe the optical window effect for radiative cooling at the MIR wavelengths. Also, sapphire substrate can be useful to control the optical absorption at MIR, i.e, if we need a transparency window extending to the longer wavelengths, $\mathrm{CaF}_{2}, \mathrm{MgF}_{2}$, $\mathrm{LaF}_{3}$ can be utilised as they reach the 7.5-to- $10 \mu \mathrm{m}$ cut off wavelengths. Quartz or fused silica are not transparent at the MIR range and strongly absorbs in that spectral range. For the propose 
of this study, the application of window for buildings, we need to block the near-IR-to-MIR light that is the heating source for the room. Hence, soda lime glass is better for that purpose. However due to reciprocity, the heat generated inside room is not leaving it by radiation through the window (it is absorbed and reflected back causing a greenhouse effect).

Figure 9 shows comprehensive simulation results for $\varepsilon_{\infty}=4.4, \omega_{\mathrm{P}}$ was changing from $1.0 \times$ $10^{14}$ to $1.0 \times 10^{16} \mathrm{rad} / \mathrm{s}, \Gamma$ is from $1.0 \times 10^{14}$ to $1.0 \times 10^{16} 1 / \mathrm{s}$. This presents analysis of effect of $\omega_{\mathrm{P}}$ and $\Gamma$ on resonances due to the ground plate. It is obvious that $\omega_{\mathrm{P}} \geq 10^{16}$ is required to obtain metasurface with optical properties useful for radiative cooling and thermal emitters. Also, a smaller $\Gamma$ is required for stronger modulation of $T$ and $R$. If the electron conductivity is too small, which means smaller $\omega_{\mathrm{P}}$ larger $\Gamma$, it is difficult to form the mirror image induced by plasmon resonance on the surface of ITO, due to the ohmic losees. Therefore optical properties becomes similar to the nanodisc structures made out of dielectric material. FDTD simulation shows separation between conventional nanodiscs and metasurfaces, that conductivity beyond $\sigma=1 \times 10^{5} \Omega . \mathrm{cm}^{-1}$ is required to obtain metasurface properties extending from visible to the MIR spectral region [40,41]. It is worth noting, that even a lower conductivity $\sigma$ at $10^{4} \Omega . \mathrm{cm}^{-1}$, larger nanodiscs, longer wavelengths, optical properties of metasurfaces become recognisable. This is due to the frequency dependence of materials parameter in Eqn. (1), where the imaginary part $\varepsilon_{2}$ becomes larger following trend for a larger $\sigma$. With increasing $\sigma$, the absorption of ITO also becomes larger and transmittance $T$ becomes negligible.

\section{Conclusion}

In this study we have realized a visible transparency and mid-infrared absorption (emission) metasurfaces for potential control of thermal radiation. Metasurfaces with ITO substrate reached $80 \%$ of transparency at visible wavelengths and their transmission band into IR-transparency window of air (or other ambient) can be engineered. Such ITO-base MIM metasurfaces placed on MIR-transparent windows can be used for radiative cooling at the designed specific spectral range (see concept in Fig. 1). The application of radiative heating and cooling of room in the buildings is now undergoing active research for reduction of electric energy consumption [42] and for agriculture greenhouse applications for sustainable energy development.

\section{References}

1. B. Zhao, C. Guo, C. A. Garcia, P. Narang, and S. Fan, "Axion-Field-Enabled Nonreciprocal Thermal Radiation in Weyl Semimetals,” Nano Lett. 20, 1923-1927 (2020).

2. M. Lim, J. Song, S. S. Lee, J. Lee, and B. J. Lee, "Surface-Plasmon-Enhanced Near-Field Radiative Heat Transfer between Planar Surfaces with a Thin-Film Plasmonic Coupler," Phys. Rev. Appl. 14 (2020).

3. F. B. Barho, F. Gonzalez-Posada, L. Cerutti, and T. Taliercio, "Heavily Doped Semiconductor Metamaterials for Mid-Infrared Multispectral Perfect Absorption and Thermal Emission,” Adv. Opt. Mater. 8 (2020).

4. A. Lenert, Z. J. Berquist, and K. K. Turaczy, "Plasmon-enhanced greenhouse selectivity for high-temperature solar thermal energy conversion,” ACS Nano 14, 12605-12613 (2020).

5. K. Yada and A. Sakurai, "Active thermal radiation control with nanoslit graphene metasurface," J. Quant. Spectrosc. Radiat. Transf. 260 (2021).

6. K. Shi, Y. Sun, Z. Chen, N. He, F. Bao, J. Evans, and S. He, "Colossal Enhancement of Near-Field Thermal Radiation across Hundreds of Nanometers between Millimeter-Scale Plates through Surface Plasmon and Phonon Polaritons Coupling," Nano Lett. 19, 8082-8088 (2019).

7. T. Cao, X. Zhang, W. Dong, L. Lu, X. Zhou, X. Zhuang, J. Deng, X. Cheng, G. Li, and R. E. Simpson, “Tuneable Thermal Emission Using Chalcogenide Metasurface," Adv. Opt. Mater. 6 (2018).

8. W. Li and S. Fan, "Nanophotonic control of thermal radiation for energy applications," Opt. Express 26, 15995 (2018).

9. R. Ma, D. Wu, Y. Liu, H. Ye, and D. Sutherland, "Copper plasmonic metamaterial glazing for directional thermal energy management," Mater. Des. 188 (2020)

10. Q. Kang, D. Li, K. Guo, J. Gao, Z. Guo, and Z. Tunable Thermal, "Tunable Thermal Camouflage Based on GST Plasmonic Metamaterial," nanomaterials (2021).

11. J. Song, S. Huang, Y. Ma, Q. Cheng, R. Hu, and X. Luo, "Radiative metasurface for thermal camouflage, illusion and messaging," Opt. Express 28, 875 (2020). 
12. Y. Zhang, C. H. Wang, H. L. Yi, and H. P. Tan, "Multiple surface plasmon polaritons mediated near-field radiative heat transfer between graphene/vacuum multilayers," J. Quant. Spectrosc. Radiat. Transf. 221, 138-146 (2018).

13. T. Kondo, S. Hasegawa, T. Yanagishita, N. Kimura, T. Toyonaga, and H. Masuda, "Control of thermal radiation in metal hole array structures formed by anisotropic anodic etching of Al," Opt. Express 26, 27865 (2018).

14. J. Yang, W. Du, Y. Su, Y. Fu, S. Gong, S. He, and Y. Ma, "Observing of the super-Planckian near-field thermal radiation between graphene sheets," Nat. Commun. 9 (2018).

15. M. Ryu, Y. Nishijima, S. Morimoto, N. To, T. Hashizume, R. Matsubara, A. Kubono, J. Hu, S. H. Ng, S. Juodkazis, and J. Morikawa, "Hyperspectral molecular orientation mapping in metamaterials," Appl. Sci. 11, 1544 (2021).

16. T. Beni, N. Yamasaku, T. Kurotsu, N. To, S. Okazaki, T. Arakawa, A. Balcytis, G. Seniutinas, S. Juodkazis, and Y. Nishijima, "Metamaterial for hydrogen sensing," ACS Sens. 4, 2389-2394 (2019).

17. T. Kumagai, N. To, A. Balcytis, G. Seniutinas, S. Juodkazis, and Y. Nishijima, "Kirchhoff's thermal radiation from lithography-free black metals," Micromachines 11, 824 (2020).

18. S. Lundgaard, S. H. Ng, Y. Nishijima, M. Mazilu, and S. Juodkazis, "Black metals: Optical absorbers," Micromachines 11, $256(2020)$.

19. Y. Nishijima, S. Morimoto, A. Balcytis, T. Hashizume, R. Matsubara, A. Kubono, N. To, R. Meguya, J. Morikawa, and S. Juodkazis, "Efficient molecular emitter," ChemRxiv p. 256 (2020).

20. Y. N. N. To, S. Juodkazis, "Detailed experiment- theory comparison of mid-infrared metasurface perfect absorbers," Micromachines 11, 409 (2020).

21. Y. Nishijima, A. Balcytis, S. Naganuma, G. Seniutinas, and S. Juodkazis, "Kirchhof's metasurfaces towards effcient photo-thermal energy conversion," Sci. Rep. 9, 8284 (2019).

22. Y. Nishijima, A. Balcytis, S. Naganuma, G. Seniutinas, and S. Juodkazis, "Tailoring metal and insulator contributions in plasmonic perfect absorber metasurfaces," ACS Appl. Nano Mater. 1, 3557-3564 (2018).

23. S. Shrestha, Y. Wang, A. C. Overvig, M. Lu, A. Stein, L. Dal Negro, and N. Yu, "Indium Tin Oxide Broadband Metasurface Absorber," ACS Photonics 5, 3526-3533 (2018).

24. K. S. Modi, J. Kaur, S. P. Singh, U. Tiwari, and R. K. Sinha, "Extremely high figure of merit in all-dielectric split asymmetric arc metasurface for refractive index sensing," Opt. Commun. 462 (2020).

25. R. Ramarajan, M. Kovendhan, K. Thangaraju, D. P. Joseph, R. R. Babu, and V. Elumalai, "Enhanced optical transparency and electrical conductivity of Ba and Sb co-doped SnO2 thin films," J. Alloy. Compd. 823 (2020).

26. M. Safari, N. P. Kherani, and G. V. Eleftheriades, "Multi-Functional Metasurface: Visibly and RF Transparent, NIR Control and Low Thermal Emissivity," Adv. Opt. Mater. (2021).

27. Z. Yan, J. Bao, X. Y. Yue, X. L. Li, Y. N. Zhou, and X. J. Wu, "Impacts of preparation conditions on photoelectric properties of the $\mathrm{ZnO}$ :Ge transparent conductive thin films fabricated by pulsed laser deposition," J. Alloy. Compd. 812 (2020).

28. T. Okatani, Y. Abe, T. Nakazawa, K. Hane, and Y. Kanamori, "Fabrication of silicon nanospheres placeable on a desired position for dielectric metamaterials in the visible region," Opt. Mater. Express 11, 189 (2021).

29. C. Lopez-Santos, D. Puerto, J. Siegel, M. Macias-Montero, C. Florian, J. Gil-Rostra, V. López-Flores, A. Borras, A. R. González-Elipe, and J. Solis, "Anisotropic Resistivity Surfaces Produced in ITO Films by Laser-Induced Nanoscale Self-organization,” Adv. Opt. Mater. 9 (2021).

30. J. H. Kim, T. Y. Seong, K. J. Ahn, K. B. Chung, H. J. Seok, H. J. Seo, and H. K. Kim, "The effects of film thickness on the electrical, optical, and structural properties of cylindrical, rotating, magnetron-sputtered ITO films," Appl. Surf. Sci. 440, 1211-1218 (2018).

31. Y. T. Li, D. T. Chen, C. F. Han, and J. F. Lin, "Effect of the addition of zirconium on the electrical, optical, and mechanical properties and microstructure of ITO thin films," Vacuum 183 (2021).

32. T. J. Hsueh, C. H. Peng, and W. S. Chen, "A transparent ZnO nanowire MEMS gas sensor prepared by an ITO micro-heater," Sensors Actuators, B: Chem. 304 (2020).

33. X. Chai, Y. Zhang, Y. Cao, L. Wu, J. Ma, Y. Liu, and L. Song, "Modulation of photoelectric properties of indium tin oxide thin films via oxygen control, and its application to epsilon-near-zero properties for an infrared absorber," J. Appl. Phys. 128 (2020).

34. D. U. Yildirim, A. Ghobadi, M. C. Soydan, O. Atesal, A. Toprak, M. D. Caliskan, and E. Ozbay, "Disordered and Densely Packed ITO Nanorods as an Excellent Lithography-Free Optical Solar Reflector Metasurface," ACS Photonics 6, 1812-1822 (2019).

35. S. Zhang, T. Wang, S. Lin, Y. Zhang, T. Tesfamichael, J. Bell, and H. Wang, "Effect of different thermo-treatment at relatively low temperatures on the properties of indium-tin-oxide thin films," Thin Solid Films 636, 702-709 (2017).

36. H. M. Ali, H. A. Mohamed, and S. H. Mohamed, "Enhancement of the optical and electrical properties of ITO thin films deposited by electron beam evaporation technique," EPJ Appl. Phys. 31, 87-93 (2005).

37. N. Wolf, D. Gerstenlauer, and J. Manara, "Modelling the spectral reflectances of miscellaneous ito coatings by using only the drude theory," J. Physics: Conf. Ser. 395, 012064 (2012).

38. Y. Nishijima, N. To, A. Balcytis, and S. Juodkazis, "Absorption and scattering in perfect thermal radiation absorber-emitter metasurfaces," ChemRxiv (2021).

39. N. Katsonis, T. Kudernac, M. Walko, S. J. van der Molen, B. J. van Wees, and B. L. Feringa, "Reversible conductance switching of single diarylethenes on a gold surface,", Adv. Mater. 18, 1397-1400 (2006).

40. S. Ray, R. Banerjee, N. Basu, A. K. Batabyal, and A. K. Barua, "Properties of tin doped indium oxide thin films prepared by magnetron sputtering," J. Appl. Phys. 54, 3497 (1983). 
41. Y. Ohhata, F. Shinoki, and S. Yoshida, "Optical properties of r.f. reactive sputtered tin-doped $\mathrm{in}_{2} \mathrm{O}_{3}$ films," Thin Solid Films 59, 255-261 (1979).

42. M. Santamouris and J. Feng, "Recent progress in daytime radiative cooling: Is it the air conditioner of the future?" Buildings 8, 168 (2018). 